\title{
Changer de rythme pour sortir de la représentation
} Pippo Delbono et Romeo Castellucci

Change of Rhythm to Break with Representation: Pippo Delbono and Romeo Castellucci

\section{Pierre Katuszewski}

\section{OpenEdition}

\section{Journals}

\section{Édition électronique}

URL : https://journals.openedition.org/clo/2034

DOI : $10.4000 /$ clo. 2034

ISSN : 2266-1816

Éditeur

INALCO

\section{Édition imprimée}

Date de publication : 1 janvier 2013

ISBN : 978-2-85831-217-7

ISSN : 0396-891X

\section{Référence électronique}

Pierre Katuszewski, «Changer de rythme pour sortir de la représentation », Cahiers de littérature orale [En ligne], 73-74 | 2013, mis en ligne le 11 mai 2015, consulté le 30 juin 2021. URL : http:// journals.openedition.org/clo/2034; DOI : https://doi.org/10.4000/clo.2034

Ce document a été généré automatiquement le 30 juin 2021.

\section{cc) (i) (3)}

Cahiers de littérature orale est mis à disposition selon les termes de la Licence Creative Commons Attribution - Pas d'Utilisation Commerciale 4.0 International. 


\title{
Changer de rythme pour sortir de la représentation
}

\author{
Pippo Delbono et Romeo Castellucci \\ Change of Rhythm to Break with Representation: Pippo Delbono and Romeo \\ Castellucci
}

Pierre Katuszewski

1 Le théâtre contemporain occidental, dans son ensemble, est un théâtre sans rituel et sans code, où la question du rythme et de ses changements ne va pas de soi. Certains types de théâtre sont en effet construits à partir d'un code rythmique. Ainsi, le théâtre nô, par exemple, pour lequel la notion de rythme est constitutive du processus de composition. Un même principe rythmique régit en effet la construction du nô: le fameux Jô/Hâ/Kyû, « ouverture, développement, finale » qui est appliqué aussi bien à la journée, à chacune des pièces, à leurs différentes parties et à chaque vers (Zeami, 1960, 24-27). Le texte doit forcément être conforme à ce code initial.

2 Le processus est donc bien différent de celui adopté majoritairement par les metteurs en scène contemporains, notamment par ceux qui composent leurs créations à partir d'un texte. Si nous pouvons supposer qu'un grand nombre d'entre eux aborde ces questions à un moment ou à un autre du travail de répétition, il en est peu qui - comme Claude Régy (Naugrette, 2008) - fondent leurs mises en scène à partir de considérations rythmiques.

3 Cependant, la question du rythme et de ses variations mérite d'être abordée. En effet, il est une catégorie de metteurs en scène qui composent leurs spectacles en proposant une succession de séquences. Bruno Tackels a récemment défini ces praticiens comme des « écrivains de plateau ». Il constate qu'

insensiblement, la mise en scène est déjà en train de devenir un métier ancien, massif encore, mais dont l'ombre portée laisse déjà entrevoir de nouvelles langues de la scène. Ceux qui les inventent occupent d'emblée la fonction d'écrivain, écrivain d'un genre particulier, dont le médium et la matière proviennent essentiellement du plateau, même si de nombreux éléments textuels peuvent 
organiquement s'y déployer. La vraie différence tient dans le fait que le texte provient

de la scène, et non du livre. (Tackels, 2009, 10)․․

Le texte est considéré comme l'ensemble de ce qui se déroule sur la scène: paroles prononcées par les acteurs, mais aussi mouvements des corps, images plastiques, etc. Les " écrivains de plateau " ne créent pas leur mise en scène à partir d'un texte préexistant : ils écrivent un spectacle du début à la fin (musique, mouvements, texte le cas échéant, lumière, etc.). Et c'est la somme de ces éléments qu'il faut s'attacher à décrypter pour saisir quelque chose de ces spectacles et plus particulièrement leur composition rythmique.

Deux exemples pris dans la création théâtrale contemporaine permettront de poser les jalons d'une analyse en termes de rythme.

6 Les spectacles de Pippo Delbono et ceux de Romeo Castellucci ${ }^{2}$ ont en commun de ne pas être construits autour d'une narration, aucun temps linéaire ne vient sous-tendre leur organisation: «On les regarde comme on écouterait des morceaux de musique. Bien davantage que dans les spectacles qui reposent sur une narration linéaire, les variations de rythme sont ici essentielles » (Tackels, 2009, 43). Le rythme s'entendant comme «tout phénomène, perçu ou agi, auquel on peut attribuer au moins deux des qualités suivantes: structure, périodicité, mouvement». Et, selon une définition restreinte : "tout phénomène, perçu ou agi, auquel on peut attribuer chacune de ces trois qualités » (Sauvanet, 1992, 238).

7 En observant la structure de certains de ces spectacles, puis en examinant quelques éléments rythmiques des séquences les composant, nous constaterons qu'une sorte de code s'établit pour chacun d'entre eux et est parfois repris d'un spectacle à l'autre, chez Pippo Delbono par exemple. Créer ainsi un spectacle à partir d'une structure rythmique et de ses variations est une façon de " codifier » le théâtre et d'établir un rapport entre l'espace scénique et l'espace du public - un rapport différent de celui établi lorsqu'il s'agit de donner à voir la mise en scène d'un texte, c'est-à-dire l'advenu d'un ou plusieurs sens par le déploiement de signes scéniques attestant d'une partition sémiologique. Il ne s'agit pas ici d'opposer le théâtre "à texte» et le théâtre "d'images", comme ce fut le cas lors de la désormais fameuse "querelle d'Avignon 2005 » (Banu et Tackels, 2005), mais de proposer une analyse des spectacles des « écrivains de plateau » détachée des méthodes classiques d'analyse de la mise en scène occidentale. Le passage par l'analyse rythmique permet en effet de s'en affranchir et d'ouvrir d'autres pistes méthodologiques.

In fine, il s'agira d'avancer des hypothèses sur ce que ces variations de rythme - et la codification qu'elles introduisent - peuvent provoquer chez les spectateurs.

\section{Structure : séquences, code et précipitation}

9 L'analyse de spectacles en études théâtrales consiste généralement à établir d'abord la liste des thèmes et des sujets traités, puis à décrire la façon dont le metteur en scène s'en est emparé afin de créer un objet esthétique ${ }^{3}$. Ici, nous évoquerons les thèmes des spectacles analysés, mais la suggestion est ensuite de sortir de ce type d'analyse, car une lecture possible serait précisément de considérer ces créations comme des propositions d'affranchissement de l'espace scénique en tant qu'espace de reproduction - fût-elle la plus abstraite qui soit - de la réalité extra-théâtrale ${ }^{4}$. Aussi, afin de décrire la structure rythmique des spectacles de Pippo Delbono et de 
Romeo Castellucci, faut-il les situer dans le contexte théâtral contemporain et examiner le rapport que chacun entretient avec l'extra-théâtralité, pour cerner la façon dont ces metteurs en scène utilisent des matériaux appartenant à l'actualité passée ou à la littérature et les modifient lors du passage à la scène.

De ce point de vue, l'analyse rythmique des spectacles serait alors un moyen - parmi d'autres - de s'affranchir d'une vision du plateau de théâtre comme espace de représentation engageant un rapport au public différent qui sera explicité en conclusion de cette analyse.

11 En effet, les spectacles de Pippo Delbono et de Romeo Castellucci appartiennent à la catégorie du théâtre postdramatique ${ }^{5}$, un théâtre qui se serait affranchi de la fable construite autour d'une intrigue avec un début, un milieu, une fin et son lot de rebondissements et, plus que cela, de la scène comme espace de représentation (au sens d'imitation ou de reproduction) d'une histoire vraisemblable ou de ce que l'on nomme communément « le réel $»^{6}$. Le théâtre postdramatique est proche de la performance en arts plastiques, c'est-à-dire d'un événement éphémère, au présent, sans faux-semblant et dont le texte la plupart du temps ne constitue pas l'élément principal. Ces spectacles sont bâtis - c'est l'hypothèse que nous allons explorer - autour d'un autre référentiel, spectaculaire et non plus narratif.

\section{Pippo Delbono : le rythme comme code}

Si les spectacles de Pippo Delbono ont pour point de départ un événement réel ou des extraits de texte, ce matériau reste un prétexte à la composition quasiment musicale ou cinématographique ${ }^{7}$ de ses spectacles. Le tremblement de terre de la ville sicilienne de Gibellina en 1968 pour Il Silenzio, la mort d'ouvriers dans une usine Thyssenkrup de Turin pour la Menzogna, sa propre maladie dans Questo Buio Feroce ne sont pas exploités comme matériaux signifiants destinés à produire du discours, mais servent à la fabrication de moments spectaculaires voués à provoquer des émotions, violentes la plupart du temps, chez les spectateurs (Tackels, 2009,51-64). Le discours essentiellement politique - n'est pourtant pas absent des spectacles, il est réservé à Pippo Delbono lui-même qui vient interrompre à plusieurs reprises les spectacles afin de disserter avec ironie et sarcasme sur l'actualité. Deux types de rapport à l'extrathéâtralité sont donc mis en œuvre: des séquences purement spectaculaires alternent avec des prises de parole " vraies ", majoritairement assumées par le metteur en scène lui-même, soit qu'il fasse référence à l'actualité ${ }^{8}$, soit qu'il présente, lors de séquences métathéâtrales, certains de ses comédiens, venant d'horizons très différents, et raconte la façon dont il les a rencontrés et dont ils ont intégré la compagnie (Delbono, 2004, 83-90).

D'un point de vue rythmique, le procédé est aisément repérable : les spectacles sont comme une succession d'images nombreuses, visibles plus ou moins longtemps, avec un rythme qui évolue au sein de chacune. Il évolue en crescendo, d'un débit lent à quelque chose de plus rapide, d'une voix basse à une voix criée, ou bien par la combinaison des deux procédés.

Par exemple, dans Il Silenzio, un acteur et une actrice effectuent sur un mouvement lent une pantomime comique avec comme fond sonore une chanson de Brigitte Bardot ("Une histoire de plage »); à la fin de la scène, ils forment une sorte de ronde de plus en plus rapide jusqu'à ce qu'ils tombent enlacés sur le sol et roulent l'un sur l'autre. 
Pendant cette dernière partie de la séquence, Pippo Delbono regarde la scène et répète de plus en plus fort une phrase: «Dis-moi que tu m'aimes » tandis que la chanson de Bardot continue d'être diffusée. Sa voix atteint le cri, le noir se fait alors sur la scène et des bruits d'éclairs et de pluie succèdent à la chanson, Delbono court sur la scène et procède à un changement de décor énergique aidé d'un technicien. Une autre séquence commence sur une musique lente de violoncelle. Delbono parcourt le plateau et lit un texte, doucement, et petit à petit sa voix devient plus forte et, une fois encore, il atteint la voix criée. Sans transition, le même comédien qui jouait dans la scène sur la chanson de Brigitte Bardot entre en déshabillé féminin, le bas du corps nu, le sexe caché entre les jambes. Il fume une cigarette et effectue quelques mouvements dansés (comme dans une boîte de nuit) sur la chanson de Dalida ( Je veux mourir sur scène»); Pippo Delbono est là, avec une lampe de poche, éclairant le comédien. Un gyrophare orange clignote, installant une atmosphère de cabaret.

Les spectateurs sont ainsi face à une alternance de séquences qui n'appartiennent pas à un récit, Delbono rompt avec un théâtre didactique et pédagogique.

L'attente n'est plus d'ordre narratif, mais uniquement spectaculaire : quelle musique va être diffusée, quel acteur va entrer en scène, comment Pippo Delbono va-t-il intervenir? Ce sont autant de questions qui provoquent un suspense spectaculaire.

En outre, les spectateurs retrouvent les mêmes procédés d'un spectacle à l'autre : les scènes se succèdent avec des motifs récurrents joués par une compagnie dont la composition varie peu d'une production à l'autre. Il y aura toujours des scènes avec un accompagnement musical qui alterneront avec des scènes parlées (en général, un acteur ou une actrice parodiant un homme politique italien), des scènes prises en charge par le metteur en scène lui-même et des scènes avec les deux acteurs en situation de handicap de la compagnie (Bobo microcéphale et Gianluca trisomique), etc.

Un code est ainsi mis en place. Les spectateurs habitués aux créations de Pippo Delbono savent qu'ils retrouveront tous ces éléments quand ils verront un nouveau spectacle. Il ne s'agit plus d'une narration, d'un temps qui serait mimétique du temps extrathéâtral, mais d'un temps spécifiquement créé par et pour le spectacle avec des motifs repérables et répétés d'une production à l'autre. Les scènes sont " attendues », le plaisir des spectateurs provient de la reconnaissance et du bon accomplissement de ce qu'ils connaissent et reconnaissent ${ }^{9}$.

19 Le rythme, entendu ici comme la succession de séquences, et la répétition au sein d'un même spectacle ou d'un spectacle à l'autre de motifs récurrents ${ }^{10}$, est constitutif de ce code spectaculaire établi par Pippo Delbono. Les spectateurs sont invités à recevoir le spectacle comme un objet non pas à lire, mais à partager :

Après la création d'un spectacle, un peu de temps m'est nécessaire pour définir, non pas le sens, mais le juste rythme, le mouvement juste, la construction. Quand on sent que la construction est terminée, le spectacle devient plus fort. Arrive un moment où on n'a plus besoin de penser à ce qu'on doit faire, puisqu'on a intégré la partition : c'est le corps, la voix qui commandent, pas la pensée. Et il faut chercher à avoir le plus d'innocence possible. (Delbono, 2004, 171).

\section{Romeo Castellucci : la précipitation}

Deux exemples pris dans le travail récent de Romeo Castellucci permettent d'examiner une autre façon de rythmer les spectacles. Dans Sul concetto di volto nel Figlio di Dio ${ }^{11}$ et dans Purgatorio ${ }^{12}$, au lieu d'avoir de multiples séquences, les spectacles sont construits 
autour de deux séquences pour le premier et trois pour le second. Entre chacune de ces parties, un changement scénographique s'opère.

\section{«Sul concetto di volto nel Figlio di Dio » : de la répétition à la précipitation}

Dans la première partie, une scène "réaliste " se répète à plusieurs reprises: un homme d'une quarantaine d'années qui vit avec son père, un vieillard incontinent, s'apprête à partir au travail. Mais au moment du départ, le père n'a pu se retenir. Son fils change sa couche tout en hurlant et pleurant. Le vieillard sanglote. Le fils hurle et le père s'excuse de sa déchéance. La scénographie est simple : d'un côté de la scène, un canapé, une table basse et quelques meubles ; au centre, une table et, de l'autre côté, un lit et un fauteuil. En fond de scène, est projeté un tableau d'Antonello da Messina représentant le visage du Christ.

Dans la seconde partie, après qu'une partie du décor a été sortie de scène, des enfants sortent des grenades et des projectiles de leurs cartables et les jettent sur l'image du Christ, ce qui n'a pas été sans provoquer de vives réactions dans les milieux intégristes. Le tableau se déforme, se déchire, et des coulures brun-rouge apparaissent avant qu'un voile noir ne vienne recouvrir le portrait; enfin, la phrase "tu es mon berger» apparaît sur l'écran puis se transforme en « tu n'es pas mon berger » (Mégevand, 2011).

Les spectateurs assistent donc à une scène se répétant plusieurs fois, à la limite du supportable tant le réalisme est suggéré, notamment par des odeurs très fortes diffusées dans la salle. Puis, Castellucci provoque un effet de surprise en brisant cette répétition de la scène entre le père et le fils : après avoir été installés dans une certaine atmosphère, les spectateurs sont littéralement "projetés » dans un autre cadre. Si la surprise provient du changement de type de narration, on passe globalement du réalisme de la scène entre le père et le fils au symbolisme de la destruction puis à la disparition de l'image du Christ, il est également possible de voir, avec ce procédé, un moyen de provoquer le spectateur en changeant le rythme de sa perception des images scéniques. Il est tout d'abord face à une action qui se répète (scène du père et du fils) crescendo, donc avec une accélération du rythme et des sentiments, ce qui se traduit par une exacerbation des émotions exprimées (les sanglots du père et les cris du fils), puis, une rupture nette intervient quand commence la séquence des enfants attaquant l'image du Christ. La rupture est d'autant plus forte que le spectateur s'est habitué à la répétition en crescendo.

\section{« Purgatorio » : ralentir pour mieux précipiter}

D'une façon comparable, le spectateur est placé en situation d'attente dans Purgatorio. Dans cette pièce, il est de nouveau face à une séquence réaliste: la scénographie présente un intérieur, tantôt un salon avec cuisine américaine, tantôt une chambre d'enfant. La mère prépare à manger à son fils qui déclare qu'il n'a pas faim. Il a mal à la tête et demande s' 'il rentre ce soir ». Un long moment plus tard, le père rentre du travail et raconte à sa femme son voyage d'affaires, « des dialogues insignifiants entre le mari et la femme racontent la banalité de leur vie quotidienne » (Mével, 2009, 62-63). Le jeu est lent et au bout de vingt minutes, les personnages nommés "Étoile 1, 2 et 3 " ne parlent plus: leurs paroles et des didascalies indiquant les mouvements qu'ils accomplissent sur la scène sont projetées sur l'écran translucide qui sépare la scène de l'espace du public depuis le début du spectacle. Ce qui déréalise ce semblant de théâtre 
naturaliste. Les spectateurs se trouvent alors mis dans une sorte d'état léthargique en raison de la lenteur et de la banalité des actions.

$\mathrm{Au}$ bout de quarante minutes de ces scènes de famille ${ }^{13}$, le fils et le père vont dans la chambre de l'enfant. Plus rien ne se passe sur la scène. Pendant cinq bonnes minutes, le père abuse de son fils, ce que les spectateurs entendent à partir d'une bande-son diffusée $^{14 .}$ Ensuite, le père revient dans le salon suivi quelques minutes plus tard par l'enfant. Tout est toujours très lent. Enfin, le fils enlace son père en lui disant: « Ne t'inquiète pas, tout est fini... tout est fini... tout est fini $»^{15}$.

Entre les deux parties de ces spectacles de Castellucci que nous venons de décrire, il y a ce que Mathieu Mével décrit comme un "précipité » créé par le changement d'images et de rythme. En effet, la première partie du spectacle est longue, lente et répétitive, alors que la seconde vient surprendre les spectateurs. Ils sont ainsi «précipités » dans la seconde partie :

À cet instant (où rien n'est montré), Castellucci précipite les choses ( « faire le vide pour qu'une précipitation soit possible ", j'emprunte la phrase à Bernard Noël qui parle de son propre travail poétique). L'inceste n'est suggéré que par la bande-son. Il y a les spectateurs qui ne supportent pas et quittent la salle, et ceux qui se laissent aller dans cette précipitation. Cette scène centrale " précipite » le réalisme de la première partie dans les visions oniriques de la seconde.

(Mével, 2009, 63).

\section{Le rythme comme façon de sortir du quotidien}

À partir de ces trois exemples, il est possible d'avancer quelques hypothèses sur la réception par les spectateurs de la construction rythmique de ces spectacles et de la manipulation de ces rythmes.

\section{Sortir du quotidien}

Dans ces exemples, les metteurs en scène prennent leur temps pour déployer leurs images, les séquences sont plutôt longues, et lentes dans le cas de Castellucci. Ainsi, en plus des ruptures de rythme indiquées plus haut, un autre changement de rythme intervient, cette fois-ci avec le quotidien en dehors du théâtre. Les spectateurs ont à s'accoutumer à un rythme qui n'est plus celui de leur vie extra-théatrale, notamment dans le rapport contemporain aux images, comme celles présentées à la télévision et dans les médias. En outre, chez Castellucci, il leur est demandé de se focaliser (il y a succession) sur une image, puis sur une seconde, voire une troisième et non plus sur leur démultiplication (contemporanéité). Ce qui diffère de l'attention quotidienne où il est possible d'être concentré sur plusieurs supports à la fois (ordinateur(s), télévision, etc.).

La plupart de ces écrivains de plateau tiennent en effet un discours sur le monde contemporain, et notamment sur la communication, l'envahissement des images, la succession rapide et aliénante des images publicitaires ou de l'actualité et en particulier à la télévision ${ }^{16}$. Ce discours n'est certes pas nouveau ${ }^{17}$, mais il est omniprésent chez ces metteurs en scène qui sont très clairs à ce sujet : ils entendent dénoncer, d'une part, la 
rapidité des images diffusées à la télévision aujourd'hui, le fameux «zapping», et, d'autre part, l'absence de communication entre les gens. Pour Castellucci :

C'est un paradoxe, mais il n'existe plus une communauté liée par le langage, le langage est complètement détruit... le langage de la communication est une maladie, la communication adopte un langage de maladie... (Mével, 2009, 62-63).

Ce discours est somme toute assez banal de nos jours, mais il trouve dans les propositions de ces écrivains de plateau une résonnance intéressante et inédite puisqu'ils en appellent aux émotions des spectateurs et à des sensations corporelles dues aux changements de rythme par rapport à leurs habitudes quotidiennes.

La comparaison avec le théâtre japonais nô du XIVe siècle est ici probante. Ainsi, une journée de nô était composée de cinq pièces, entrecoupées de quatre intermèdes (Kyôgen), avec un ralentissement du rythme de la première à la troisième, la pièce la plus lente de la journée et la reprise d'un rythme plus rapide pour les deux dernières. René Sieffert, grand spécialiste occidental de ce théâtre, écrit que "même pour un amateur éprouvé, la troisième pièce de la journée constitue une rude épreuve dont il sort ravi, mais mentalement courbaturé » (Sieffert, 1960, 26).

Donc, il y a changement de rythme par rapport à ce que vivraient les spectateurs en dehors du théâtre, mais aussi par rapport au début du spectacle lui-même. Ce processus est comparable avec ce qui se passait pour le théâtre romain antique, puisque le rituel des jeux, au sein desquels avaient lieu les spectacles de théâtre, proposait un temps suspendu aux spectateurs, un temps différent du temps civique, un temps pendant lequel ils se détendaient afin de bien accomplir le rituel religieux (Dupont et Letessier, 2011). Les spectateurs du théâtre contemporain, confrontés à ces spectacles qui n'ont pas pour but de représenter la réalité extra-théâtrale, ne seraient-ils pas amenés à faire une expérience du même genre?

\section{Le rythme, déclencheur d'émotions}

34 Les spectateurs entrent dans une autre sphère, celle d'un théâtre construit autour d'un rythme déréalisant celui de l'en-dehors du théâtre et jouant sur l'émotion. Nous passerions d'une transmission verticale, du metteur en scène au public, à un partage horizontal : c'est de la rencontre entre les acteurs et les spectateurs que naissent les émotions - c'est à partir de l'agencement des rythmes et des changements de rythme que les metteurs en scène établissent un contact entre la scène et la salle :

Il est très important d'être avec le public, de se placer à un moment donné de son côté, c'est-à-dire de ne pas lui imposer un personnage ou une action, mais d'arriver sans défense, avec toute sa fragilité, son incertitude et de tout donner à l'œuvre. Là, l'œuvre devient plus grande que toi. Il y a des moments où je ne sais plus rien du spectacle et je ne veux pas commencer à réfléchir parce que cela reviendrait à édifier un mur qui s'interposerait entre moi et cet acteur fondamental qu'est le public. (Delbono, 2004, 151).

Commentant le théâtre de Castellucci, Mathieu Mével évoque explicitement l'émotion :

Le théâtre de Castellucci consiste à agencer un lieu (scénographie, objet, lumière) pour accueillir les images dans leur devenir, dans leur durée. Le metteur en scène y est le régisseur (selon la terminologie allemande) ou le magicien. Maître des effets et des durées, il manipule les lumières, les durées, les sons (les techniques), et les émotions. (Mével, 2009, 62).

Et dans une interview à la revue Théâtre/Public, Castellucci déclare : 
Vous avez dit que parfois il était nécessaire de ne pas comprendre.

La compréhension n'est pas quelque chose qui se joue seulement au niveau de l'intellect, je crois qu'un spectateur doit être impliqué et secoué avec tout son corps et que la connaissance passe aussi sur la peau parce que la communication, tout d'abord, est une sensation et donc il y a une communication corticale, animale, sensorielle. [...] Ce qui compte surtout, c'est l'émotion. (Castellucci, 2009).

C'est un corps éprouvé qui ressort du théâtre, éprouvé tout d'abord par le ralentissement du rythme quotidien - entre le rythme social et le rythme particulier du moment spectaculaire - puis par des changements de rythme au sein même des spectacles, par exemple avec des chocs, des « précipités » chez Castellucci.

Pippo Delbono fait aussi très souvent allusion à l'émotion. Par exemple, quand il évoque ses années d'apprentissage à l'Odin Teatret : « Ma conception de l'acteur avait évolué, je m'intéressais avant tout aux qualités de présence sur le plateau, à la capacité des acteurs à m'émouvoir » (Delbono, 2004, 5). Dans le même ouvrage, il explique que, pour lui :

La composition est un parcours d'émotions, un voyage partagé avec le public. Un critique a dit que ce que je savais faire de mieux, c'était conduire le public à travers différentes émotions. Dans ce voyage qu'est le spectacle, il faut aller dans l'émotion, mais aussi en sortir par moments, sinon on la refuse. C'est un peu le principe que j'applique en tant que metteur en scène: passer par différentes couleurs, différentes tonalités, des respirations, des ruptures. C'est un peu difficile à expliquer, c'est lié à des nécessités intérieures. En même temps, il faut conserver une distance par rapport à ce qui se passe sur le plateau, car même quand il y a de la violence ou de la tendresse sur scène, c'est toujours un jeu. (Delbono, 2004, 162).

La construction rythmique, introduisant un code, au sein même d'un spectacle ou d'un spectacle à l'autre, apparente ces spectacles aux théâtres du jeu, comme le théâtre antique ou les théâtres orientaux tels que le kathakali, le théâtre balinais ou le nô japonais. Ces théatres de l'instant et du rassemblement, puisqu'il s'agit de créer une communauté composée des acteurs et des spectateurs, suspendent le temps quotidien et offrent l'entrée dans un temps du jeu et de l'émotion ${ }^{18}$.

\section{Le choc émotionnel comme « soin » esthétique}

40 Le théâtre contemporain occidental en général n'est pas un théâtre codifié. Pippo Delbono, comme Romeo Castellucci, en construisant leur spectacle à partir d'une composition rythmique, introduisent une forme de codification et proposent un processus de sortie de la représentation au sens aristotélicien d'imitation.

41 Cette phase de codification passe donc par l'éloignement d'un théâtre de l'imitation et par la création de codes comme cette succession de séquences chez Delbono ${ }^{19}$ ou une composition en deux ou trois séquences chez Castellucci. Ils créent ainsi un rythme l'essentiel étant de faire sortir les spectateurs de leur quotidienneté, c'est là que se situerait un premier changement de rythme qui permettrait de rendre disponibles les spectateurs pour recevoir des émotions et la beauté plastique d'un objet esthétique composé de ces successions de séquences.

42 C'est à partir de l'établissement de ces rythmes qu'ils introduisent des ruptures «provoquant» les spectateurs. Dans les exemples pris ici dans le théâtre contemporain, on peut noter une sorte d'emboîtement dans les changements de rythme : après avoir fait pénétrer les spectateurs dans un rythme différent du temps 
quotidien, les metteurs en scène jouent sur les variations au sein même de leur création afin de précipiter des chocs esthétiques provoquant des émotions chez les spectateurs.

Pour conclure, on peut interroger la présence, chez Castellucci ${ }^{20}$, d'images très fortes (le père incontinent, le viol) : est-ce par volonté de choquer et de faire réfléchir le spectateur sur les aléas ou la violence de la vie ? Certes, certains peuvent s'identifier à ce qui se passe sur la scène et se sentir agressés (de nombreux spectateurs sortent au milieu des spectacles de Castellucci et de Pippo Delbono), mais ne peut-on pas retourner le point de vue ? En poursuivant la comparaison évoquée plus haut avec le théâtre nô et les courbatures du corps à la vue de spectacles très lents, ou bien avec le théâtre grec antique, où les histoires des tragédies, plus horribles les unes que les autres, n'étaient que les prétextes à une composition musicale provoquant des émotions fortes, il ne s'agirait pas forcément de renvoyer à l'extra-théâtralité, mais d'éprouver les corps, de provoquer des émotions à partir d'images fortes qui ne seraient plus que des " prétextes », justement, à émotion.

Dans le cas du spectacle tragique grec, Florence Dupont parle d'identification émotionnelle des spectateurs avec les choreutes. Il s'agit de ressentir des émotions extrêmes, provoquées par les chants de deuil et les danses du chœur et la musique stridente de l'aulos (Dupont, 2009, 88). Éprouver ces émotions et réussir le rituel spectaculaire est une manière d'être en paix avec les dieux et donc de maintenir l'équilibre de la communauté. En d'autres termes, le rituel extrait l'ensemble des individus du quotidien afin de leur procurer du plaisir (ainsi qu'aux dieux destinataires des chants du chœur).

Par analogie, nous dirons que les changements de rythme, mis en œuvre par les metteurs en scène contemporains évoqués ici, sont une façon de prendre en compte le spectateur et de lui dispenser une sorte de « soin » esthétique : on prend soin de lui en lui laissant le temps de ressentir des émotions fortes ${ }^{21}$, ce qui est aussi un moyen de recentrer le corps des spectateurs sur lui-même et de recréer un espace communautaire temporaire.

\section{BIBLIOGRAPHIE}

BANU, Georges, TACKELS, Bruno (éds), 2005, le Cas Avignon 2005. Regards critiques, Vic-la-Gardiole, L'Entretemps.

BOUVET, Bruno, Hommes sans dieu, Webthea, $1^{\text {er }}$ août 2011, consulté le 9 avril 2015.

CASTELLUCCI, Romeo, 2009, Une nouvelle séquence théâtrale européenne, Théâtre/Public, nº 194.

Delbono, Pippo, 2004, Mon théâtre, Arles, Actes Sud.

Dossier pédagogique du spectacle « la Divine Comédie ", Scérén, $\mathrm{n}^{0} 51$, juillet 2008, consulté le 9 avril 2015.

DUPONT, Florence, LETESSIER, Pierre, 2011, le Théâtre romain, Paris, Armand Colin. 
DUPONT, Florence, 2009, la musique tragique, in Sophocle, Antigone, traduit par Florence Dupont, Paris, L'Arche, p. 87-94.

KATUSZEWSKI, Pierre, 2011, Ceci n'est pas un fantôme. Essai sur les personnages de fantômes dans les théâtres antique et contemporain, Paris, Kimé.

LEHMANN, Hans-Thies, 2002, Le Théâtre postdramatique, Paris, L'Arche.

MÉGEVAND, Matthieu, 2011, Des intégristes catholiques perturbent « Sur le concept du visage du fils de Dieu », le Monde des religions, 24 octobre 2011, consulté le 9 avril 2015.

MÉVEL, Mathieu, 2009, « la Fête du refus », « Une nouvelle séquence théâtrale européenne », Théâtre/Public, nº 194, p. 61-66.

NAUGRETTE, Catherine, 2008, Pour une métaphysique de la lenteur, in Marie-Madeleine MervantRoux (éd.), Claude Régy, Paris, CNRS éditions.

PASOLINI, Pier Paolo, 2003, Contre la télévision, in Pier Paolo Pasolini, Contre la télévision, Besançon, Les Solitaires intempestifs, p 25-46. Trad. de l'italien par Michel Caroline et Joubert-

Laurencin Hervé [ $1^{\text {re }}$ éd., 1966].

PAVIS, Patrice, 2012, l'Analyse des spectacles, Paris, Armand Colin. [1 $1^{\text {re }}$ éd, 2005].

SARRAZAC, Jean-Pierre, 2000, Critique du Théâtre : de l'utopie au désenchantement, Paris, Circé.

SAUVANET, Pierre, 1992, le Rythme encore une définition, in Jean-Jacques Wunenburger (éd.), les

Rythmes. Lectures et théories, Paris, L'Harmattan.

SIEFFERT, René, 1960, Introduction, in Zeami, 1960, la Tradition secrète du nô, Paris, Gallimard. Trad. et commentaires de René Sieffert.

TACKELS, Bruno, 2009, Pippo Delbono, Besançon, Les Solitaires intempestifs.

TACKELS, Bruno, 2007, Rodrigo Garcia, Besançon, Les Solitaires intempestifs.

WUNENBERGER, Jean-Jacques (éd.), 1992, les rythmes : lectures et théories, Paris, L'Harmattan.

ZEAMI, la Tradition secrète du nô, 1960, Paris, Gallimard. [Trad. et commentaires de René Sieffert].

\section{NOTES}

1. L'auteur souligne.

2. D'autres expériences auraient pu être analysées dans le cadre de cet article, on pense ici à Jan Fabre, Joseph Nadj, Rodrigo Garcia, Pina Bausch ou encore François Verret.

3. La description est bien évidemment ici schématique, pour une vue d'ensemble sur les différentes méthodes d'approche des spectacles, cf. PAvis (2012).

4. Nous avons proposé ce type d'analyse dans KATUSZEWSKI (2011). L'analyse était alors centrée sur les personnages de fantômes dans le spectacle tragique antique (romain et grec) puis chez quelques auteurs de théâtre contemporain. Nous proposons ici d'élargir l'analyse en termes de spectacles aux créations spectaculaires de Pippo Delbono et Romeo Castellucci. La comparaison avec le spectacle antique tel qu'il est abordé depuis quelques années (cf. les travaux de Florence Dupont, Pierre Letessier, Emmanuelle Vallette-Cagnac, William Marx, etc.) et les outils méthodologiques de 
disciplines comme l'ethnopoétique nous semblent ainsi plus adéquats par rapport à la réalité des spectacles d'une grande partie du théâtre contemporain.

5. La catégorie du théâtre postdramatique est complexe et multiple. Elle est utilisée ici dans son acception la plus large. Pour une analyse détaillée, cf.LeHMANN (2002).

6. "Le théâtre postdramatique est un théâtre qui exige "un événement scénique qui serait, à tel point, pure présentation, pure présentification du théâtre qu'il effacerait toute idée de reproduction, de répétition du réel" » (SARRAZAC, 2000, 63).

7. «En ce qui concerne le montage, je compose des séquences d'images qui peuvent fonctionner de manière autonome. Chacune est terminée, chacune a un sens en soi.» (DELBONO, 2004, 105).

8. Nous renvoyons ici à ses interventions à propos du président du Conseil italien Silvio Berlusconi dans la plupart de ses spectacles, par exemple.

9. Nous formulons cette hypothèse en comparant le processus à celui des théâtres antiques d'une part et à partir de notre expérience de spectateur d'autre part (KATUSZEWSKI, 2011).

10. On pense ici par exemple à la fameuse danse de Pippo : "On la retrouve, déclinée, dans ces moments où il s'électrise, comme traversé par un flux surpuissant qui s'empare de lui, agité d'un tremblement qui semble le soulever de terre. Dans chacun de ses spectacles, elle apparaît, une fois, voire plusieurs, la danse de Pippo» (TACKELS, 2009, 74).

11. "Sur le concept du visage du fils de Dieu», spectacle créé en 2010 au festival RomaEuropa à Rome.

12. Deuxième spectacle du tryptique «la Divine comédie», créé pour la Cour d'honneur du Palais des papes en juillet 2008 dans le cadre du festival d'Avignon.

13. Pour le détail de la description du spectacle,cf. le dossier pédagogique du spectacle, p. 17-18, consulté le 29 mars 2013.

14. "C'est la scène de l'inceste entre le père et son fils. On entend des cris. On est seuls face à la scène vide pendant 5 minutes. À cet instant (où rien n'est montré) » (MÉVEL, 2009, 63).

15. Puis, le décor change et deux autres longues parties vont se succéder, cf. ibid.

16. On peut citer Rodrigo Garcia évoquant son spectacle "After sun » (2001): «Le concept qui structure le spectacle est la question des médias, et en particulier celle de la télévision. Je voulais parler de ce qui se passe aujourd'hui, de cette télé-poubelle qui me dégoûte et me fait sortir de mes gonds. Et pour en parler, il fallait que je trouve un langage très fort, avec des mots qui soient à la hauteur de mon dégoût " (TACKELS, 2007, 67).

17. Pier Paolo Pasolini écrivait déjà en 1966 un opuscule intitulé "Contre la télévision ", traduit récemment en français.

18. Ce qui fonctionne chez Pippo Delbono si l'on en croit Bruno TACKels : «C'est ce qui explique la force populaire que génèrent ses spectacles, qui tourne parfois à de la ferveur. Certains spectateurs sortent dans de drôles d'états, mutiques, en larmes, bouleversés par ce qui leur est arrivé. Ces spectacles touchent des zones très profondes de l'être humain. Certains ne veulent pas les voir, ce qui produit inévitablement une division, un clivage profond dans le public. On a vu des couples se déchirer autour de leur façon de recevoir les spectacles de Pippo Delbono!» $(2009,45)$. L'auteur souligne. 
19. Dans «Inferno » de Castellucci, il est possible de faire une analyse comparable.

20. Mais également dans la plupart des spectacles de Pippo Delbono.

21. La récente création de Rodrigo Garcia, «Golgota Picnic », est à ce titre emblématique : après une première partie énergique, foisonnante et provocatrice, la seconde voit un musicien nu jouer en entier une pièce de Haydn. Après la provocation de la première partie, il est laissé le temps aux spectateurs d'apprécier ce concert.

\section{RÉSUMÉS}

Les metteurs en scène contemporains Pippo Delbono et Romeo Castellucci composent certains de leurs spectacles à partir d'un montage de séquences et non pas à partir d'une narration, rompant ainsi avec le théâtre dramatique. Le rythme est alors constitutif de leur création. Il s'agira ici de décrire les procédés rythmiques mis en œuvre par ces deux metteurs en scène, puis d'examiner les impacts que ces changements de rythme peuvent avoir sur les spectateurs. On observera ainsi comment un premier changement de rythme est mis en place, entre le temps quotidien des spectateurs et le temps du spectacle, puis comment les manipulations du rythme provoquent des "précipitations » esthétiques. Quelques hypothèses seront proposées quant à la signification pragmatique de ces changements de rythme. En comparaison avec les théâtres antiques et orientaux, nous analyserons ces variations comme autant de façons de susciter des émotions esthétiques chez les spectateurs.

The contemporary directors Pippo Delbono and Romeo Castellucci conceive some of their shows as a montage of sequences and not as a narrative form of theater. Yet, they break up with a dramatical theater. The rhythm is a constitutive element of their creation act. This article aims to describe the rhythmic processes used by these two directors, and to examine the impact that these changes of ryhthm can have on the audience. First, one can observe how a change of rhythm is established between the time of everyday life and the time of the show, then, how the manipulation of rhythm may produce some sort of aesthetic "precipitations". Some hypotheses are proposed on the pragmatic significance of these changes of rhythm. In comparison with the ancient and oriental theaters, this article will analyse these changes as ways to produce aesthetic emotions from the perspective of the audience.

\section{INDEX}

Thèmes : théâtre

Keywords : Delbono Pippo (1959-), Castellucci Romeo (1960-), Theatre, Aesthetic, Emotion, Codification

Index géographique : Italie

Mots-clés : Delbono Pippo (1959-), Castellucci Romeo (1960-), théâtre, esthétique, émotion, codification 
AUTEUR

PIERRE KATUSZEWSKI

Université Bordeaux Montaigne, Laboratoire CLARE/ARTES (EA 4593) 